\title{
An Invitation to Combinatorial Tropical Geometry
}

\author{
Ivana Protrka \\ Faculty of Mining, Geology and Petroleum Engineering, University of Zagreb \\ Pierottijeva 6, 10000 Zagreb, Croatia \\ e-mail: ivana.protrka@rgn.hr
}

\begin{abstract}
This paper addresses an overview on the recent development of the area known as the tropical geometry. In particular we point out combinatorial aspect of the field. After a brief introduction to tropical algebra we discuss tropical hypersurfaces and applications in enumerative geometry.

Keywords: tropical geometry, tropical curve, Gromov-Witten invariant, Newton subdivision
\end{abstract}

MSC: 14A99, 14N10, 14T05, 52B20

\section{$1.1 \quad$ Introduction}

The tropical geometry is a very new area in mathematics, that is in essence closely related to algebraic geometry. While rapid development of the area begins with this century, its leading ideas had appeared in earlier works of G. M. Bergman and others. Recent significant contribution to the theory has been made by G. Mikhalkin in particular within the paper that can be understand as foundation of enumerative algebraic geometry (see [17, 18, 19, 21]).

It is worth mentioning that the attribute "tropical" has no any intuitive meaning related to the theory but was coined by French mathematicians in honor of the Brazilian

DOI: https://doi.org/10.5592/CO/CCD.2016.05 
mathematician Imre Simon, who contributes to the field [24]. In what follows we are firstly concerned with tropical arithmetic and its relations to classical arithmetic. Then we give an example of tropical geometry and combinatorial mathematics. Besides we define tropical curves and correlation with enumerative algebraic geometry.

We also presents recent results of Maclagan and Sturmfels (see [16]) and Mikhalkin (see [19]). For further introduction to tropical geometry one can look at references $[2,6,10,14,23,25,26,27]$. A more advanced reader may refer to [3, 15, 20].

As an introduction to the field we present basic ideas and facts of tropical algebra. The set of tropical numbers is defined as $\mathbb{T}=\mathbb{R} \cup\{\infty\}$ whereas operations of tropical addition and multiplication on $\mathbb{T}$ are defined as

$$
\begin{gathered}
x \oplus y:=\min (x, y) \\
x \odot y:=x+y,
\end{gathered}
$$

with the usual conventions when one of the operands is $\infty$ [16]. For example, we have $3 \oplus 5=3,3 \odot 5=8$. The set $\mathbb{T}$ equipped with these operations form a tropical semiring $(\mathbb{R}, \oplus, \odot)$ or the min-plus algebra. In this triple one can replace the operation of minimum by maximum to get the isomorphic max-plus algebra. Many of axioms from arithmetic remain also in tropical algebra. As it is the case in classical algebra, we abbreviate multiplication $x \odot y$ by $x y$.

Having in mind that zero is the neutral element in respect to multiplication, for the coefficients in Pascal triangle we have

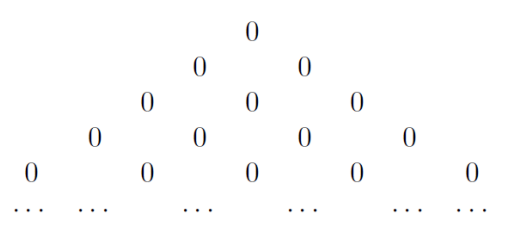

When applying the binomial theorem on the third row of the triangle we get

$(x \oplus y)^{3}=(x \oplus y) \odot(x \oplus y) \odot(x \oplus y)=0 \odot x^{3} \oplus 0 \odot x^{2} y \oplus 0 \odot x y^{2} \oplus 0 \odot y^{3}=x^{3} \oplus x^{2} y \oplus x y^{2} \oplus y^{3}$

and furthermore

$$
(x \oplus y)^{3}=x^{3} \oplus y^{3} .
$$


Clearly, in classical arithmetic we have

$$
3 \cdot \min \{x, y\}=\min \{3 x, 2 x+y, x+2 y, 3 y\}=\min \{3 x, 3 y\}
$$

for every $x, y \in \mathbb{R}$. A simple generalization of this statement give as a tropical version of the binomial theorem,

$$
(x \oplus y)^{n}=x^{n} \oplus y^{n}, n \in \mathbb{N} .
$$

Over the tropical semiring, we can define a linear algebra operations of adding and multiplying vectors and matrices in the usual way. For instance,

$$
\left(\begin{array}{ll}
0 & 1 \\
2 & 3
\end{array}\right) \odot\left(\begin{array}{ll}
4 & 5 \\
6 & 7
\end{array}\right)=\left(\begin{array}{ccc}
0 \odot 4 \oplus 1 \odot 6 & 0 \odot 5 \oplus 1 \odot 7 \\
2 \odot 4 \oplus 3 \odot 6 & 2 \odot 5 \oplus 3 \odot 7
\end{array}\right)=\left(\begin{array}{ll}
4 & 5 \\
6 & 7
\end{array}\right) .
$$

We let $x_{1}, x_{2}, \ldots, x_{n}$ be elements of $(\mathbb{T}, \oplus, \odot)$. Then one can define both monomials and polynomials in these variables on the tropical algebra. A tropical polynomial $p$ in $n$ variables is defined as the function $p: \mathbb{R}^{n} \rightarrow \mathbb{R}$,

$$
p\left(x_{1}, \ldots, x_{n}\right)=a \odot x_{1}^{i_{1}} x_{2}^{i_{2}} \cdots x_{n}^{i_{n}} \oplus b \odot x_{1}^{j_{1}} x_{2}^{j_{2}} \cdots x_{n}^{j_{n}} \oplus \cdots,
$$

with $a, b, \ldots \in \mathbb{R}, i_{1}, j_{1}, \ldots \in \mathbb{Z}$. According to the definition of tropical addition and multiplication we also have

$$
p\left(x_{1}, \ldots, x_{n}\right)=\min \left(a+i_{1} x_{1}+\cdots+i_{n} x_{n}, b+j_{1} x_{1}+\cdots+j_{n} x_{n}, \ldots\right) .
$$

The following Lemma 1 tell us that a graph of tropical polynomial is consisted by linear functions.

Lemma 1 Tropical polynomials in $n$ variables $x_{1}, \ldots, x_{n}$ are linear piecewise concave functions on $\mathbb{R}^{n}$ with integer coefficients.

The values of arguments of tropical polynomial function $p$ in the breakpoints where $p$ fails to be linear are zeros of $p$, the so-called hypersurface $V(p)$. As a concrete example of a tropical polynomial in one variable we consider

$$
\begin{aligned}
p(x) & =3 \odot x^{3} \oplus 1 \odot x^{2} \oplus 2 \odot x \oplus 4 \\
& =\min (3+3 x, 1+2 x, 2+x, 4) .
\end{aligned}
$$


The graph of this function consists of four lines: $y=3 x+3, y=2 x+1, y=x+2$ and $y=4$ (see Figure 1) and related hypersurface is $V(p)=\{-2,1,2\}$. The fundamental theorem of algebra holds true also in tropical algebra, thus tropical polynomial function can be whiten as a tropical product of linear functions. In this particular example zeros are $-2,1,2$ so we have

$$
p(x)=3 \odot(x \oplus(-2)) \odot(x \oplus 1) \odot(x \oplus 2) .
$$

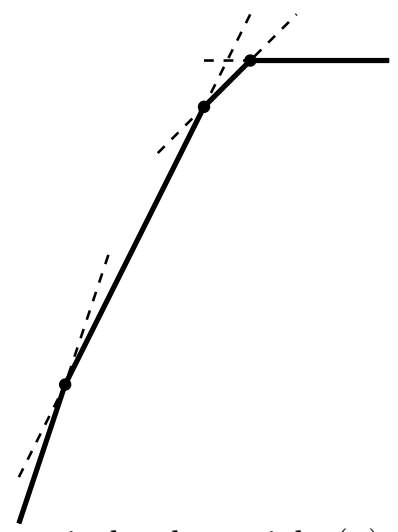

Figure 1: Graph of the tropical polynomial $p(x)=3 x^{3} \oplus 1 x^{2} \oplus 2 x \oplus 4$.

\subsection{Shortest path, tropical distance and volume}

We let $G$ denote a directed graph with $n$ nodes that are labeled by $1,2, \ldots, n$. Every directed edge $(i, j)$ in $G$ has an associated length $d_{i j} \in \mathbb{R}_{\geq 0}$. If $(i, j)$ is not an edge of $G$ then we set $d_{i, j}=+\infty$. We represent the weighted directed graph $G$ by its $n \times n$ adjacency matrix $D_{G}$,

$$
D_{G}=\left(\begin{array}{cccc}
0 & d_{1,2} & \cdots & d_{1, n} \\
d_{2,1} & 0 & \cdots & d_{2, n} \\
\vdots & \vdots & \ddots & \vdots \\
d_{n, 1} & d_{n, 2} & \cdots & 0
\end{array}\right)
$$

Off-diagonal entries are the edge lengths $d_{i j}$. The matrix $D_{G}$ need not be symmetric. Now, consider the $n \times n$ matrix which is obtained from topically multiplying the given adjacency matrix $D_{G}$ with itself $n-1$ times

$$
D_{G}^{\odot n-1}=D_{G} \odot D_{G} \odot \cdots \odot D_{G} .
$$


The problem of finding shortest paths in a weighted directed graph $G$ is solved in the following Lemma 2.

Lemma 2 Let $G$ be a weighted directed graph on $n$ nodes with $n \times n$ adjacency matrix $D_{G}$. The entry of the matrix $D_{G}^{\odot n-1}$ in row $i$ and column $j$ equals the length of a shortest path from node $i$ to node $j$ in $G$.

For $r \geq 0$, a recursive formula for the length of a shortest path from node $i$ to node $j$ which uses at most $r$ edges in $G$ we can write in the following way:

$$
d_{i j}^{(r)}=\min \left\{d_{i k}^{(r-1)}+d_{k j}: k=1,2, \ldots, n\right\} .
$$

This recursion is used in the Floyd-Warshall algorithm. This algorithm is known as the fastest algorithm for finding shortest paths in a weighted graph. For us, running that algorithm means performing the matrix multiplication

$$
D_{G}^{\odot r}=D_{G}^{\odot r-1} \odot D_{G} \text { for } r=2, \ldots n-1 .
$$

In this section we will still define tropical distance and tropical volume. For more see $[4,5]$. For two points $v, w \in \mathbb{T}^{d}$ the tropical distance is the number

$$
\begin{aligned}
\operatorname{tdist}(v, w) & :=\max \left\{\left(v_{i}-w_{i}\right) \mid i \in[d]\right\}-\min \left\{\left(v_{i}-w_{i}\right) \mid i \in[d]\right\} \\
& =\max _{i, j \in[d]}\left|v_{i}-w_{i}+w_{j}-v_{j}\right|
\end{aligned}
$$

This number is given in $[4,5]$ and he is the Euclidean distance in the tropical setting.

Let $A=\left(a_{i j}\right) \in \mathbb{T}^{d \times d}$ be a square matrix. Tropical volume of $A$ is given as the expression

$$
\operatorname{tvol} A:=\left|\bigoplus_{\sigma \in \operatorname{Sym}(\mathrm{d})} \sum a_{i, \sigma(i)} \quad-\bigoplus_{\tau \in\left(\operatorname{Sym}(\mathrm{d})-\sigma_{o p t}\right)} \sum a_{i, \tau(i)}\right|
$$

where $\sigma_{o p t}$ is a permutation for which $\sum a_{i, \sigma_{o p t}(i)}$ coincides with the tropical determinant of $A$. Tropical determinant is defined in the following way

$$
\operatorname{tropdet}(A):=\bigoplus_{\sigma \in S_{n}} x_{1 \sigma(1)} \odot x_{2 \sigma(2)} \odot \cdots \odot x_{n \sigma(n)} .
$$




\subsection{Tropical curves in $\mathbb{T}^{2}$}

In the introduction we consider a graph of a tropical polynomial in one variable and have seen that such a graph is a collection of linear concave functions. Naturally an extension of this notion within polynomials of any degree and any number of variables lead to tropical curves. For the purpose to have conceptually simpler definitions and examples here we restrict discussion to curves in $\mathbb{T}^{2}$ instead of $\mathbb{T}^{n}$. In order to define tropical curve, we must first define tropical hypersurface we mentioned in the introduction. Tropical hypersurface $V(p)$ of $p$ is the set of all points $w \in \mathbb{T}^{2}$ at which this minimum is attained at least twice. Equivalently, a point $w \in \mathbb{T}^{2}$ lies in $V(p)$ if and only if $p$ is not linear at $w$. Plane tropical curve is tropical hypersurface $V(p)$ of a polynomial in two variables

$$
p(x, y)=\bigoplus_{(i, j)} c_{i j} \odot x^{i} \odot y^{j} .
$$

A tropical line of a 2-variable polynomial $p$ is a hypersurface $V(p)$ of the first degree $p$,

$$
p(x, y)=a \odot x \oplus b \odot y \oplus c=\min (a+x, b+y, c),
$$

with $a, b, c \in \mathbb{R}$. This tropical line consists of three semi-lines with source in $(c-a, c-b)$ where directions of lines are north, east and south-west i.e. they are defined by relations $x=c-a \leq y, y=c-b \leq x$ and $y=x$, respectively. Figure 2 a) depicts such a line. In the same fashion one can study tropical quadratic curves as well as the other higher order curves. In general, tropical curve defined by $p(x, y)$ is a graph having defined the weight function on the edges, and this function is defined by means of the polynomial degree and the coefficients [2].

As an even more illustrative example let us consider a quadratic curve, defined by the polynomial $p(x, y)=a \odot x^{2} \oplus b \odot x y \oplus c \odot y^{2} \oplus d \odot y \oplus e \oplus f \odot x$. To draw the related curve one have to take into account 15 constraints arising from the definition of polynomial, which give the curve having three segments and six semi-lines. The Figure $2 \mathrm{~b})$ shows a tropical conic defined by

$$
p(x, y)=2 \odot x^{2} \oplus x y \oplus 2 \odot y^{2} \oplus x \oplus(-1) \odot y \oplus 3 .
$$

The following Lemma 3 summarizes the salient features of a plane tropical curve.

Lemma 3 The curve $V(p)$ is a finite graph that is embedded in the plane $\mathbb{R}^{2}$. It has both bounded and unbounded edges, all edge slopes are rational, and this graph satisfies a balancing condition around each node. 

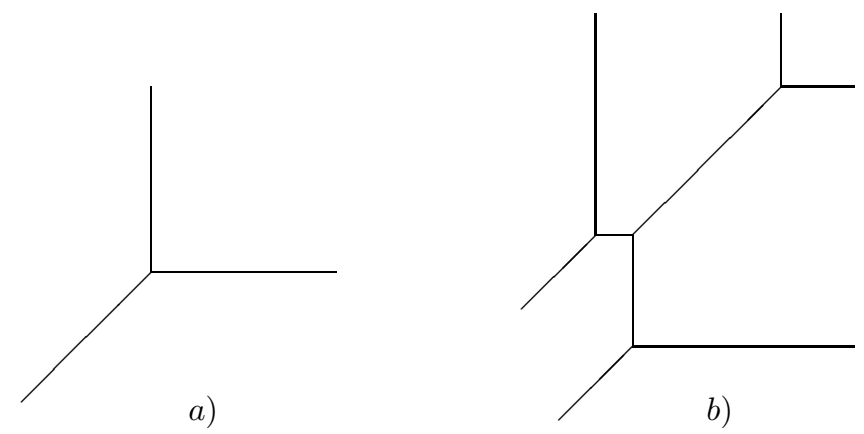

Figure 2: A tropical line and a tropical quadratic curve.

Below we give another way we can describe the tropical curves. The Newton polygon of the implicit equation $f(x, y)$ is the convex hull in $\mathbb{R}^{2}$ of all points $(i, j) \in \mathbb{Z}^{2}$ such that $x^{i} y^{j}$ appears with non-zero coefficient in the expansion of $f(x, y)$.

Lemma 4 We let $p$ is a tropical polynomial. Then $V(p)$ is a planar graph which is a dual of regular subdivision of the Newtonian polygon Newt $(p)$.

The unbounded rays of a tropical curve $V(p)$ are perpendicular to the edges of the Newton polygon. The Figure 3 a) shows a subdivisions of the Newton polygon and the Figure $3 \mathrm{~b}$ ) shows belongs to the tropical biquadratic curve.

Let consider tropical curves having Newtonian polygon being triangle with vertices $(0,0),(0, d)$ and $(d, 0)$. These curves we shall call curves of the degree $d$. The curve of the degree $d$ has $d$ semi-lines, taking into account multiplicity (the weights of edges), which are perpendicular to each of three edges of the Newtonian triangle [16, 22]. There are some facts known about intersection of two curves of a certain degree. We have it by the following Bézout's theorem (see [1,9]).

Theorem 5 (Bézout) We let $C$ and $D$ be two tropical curves of the degrees $c$ and $d$ in $\mathbb{R}^{2}$. If these curves intersect transversely then the number of intersecting points, counted with multiplicity, is equal to cd.

On the other hand, it is possible that the two curves have no neither transversal nor finite intersection. This possibility is called stable intersection and more details on it one can find at [16]. 


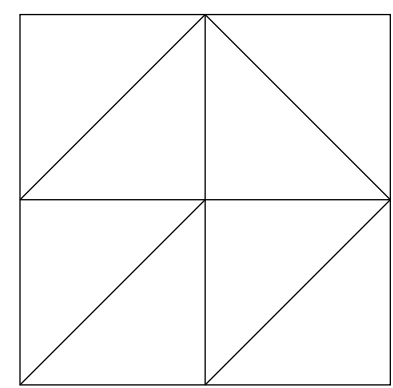

a)

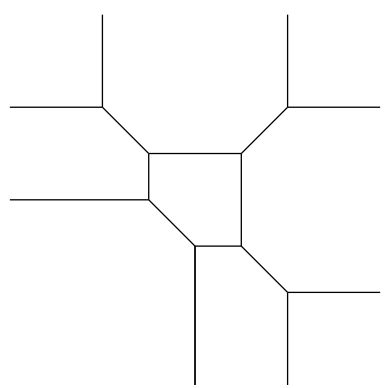

b)

Figure 3: Subdivisions of a Newton polygon and the tropical biquadratic curve

\subsection{Gromov-Witten invariants}

A complex algebraic curve is the set of zeros of a homogeneous polynomial $P(x, y, z)=$ 0 in the complex projective space $\mathbb{P}^{2}$. The degree of a complex algebraic curve is simply the degree of the polynomial $P(x, y, z)[7]$.

The central subject of the enumerative geometry is to determine Gromov-Witten invariants. This invariant counts how many of complex algebraic curves of a given degree and genus are incident with certain number of points. It is proven by G. Mikhalkin that complex algebraic curves can be replaced by tropical curves in that sense and he used this fact to derive combinatorial expression for the number of curves in the tropical case $[8,12,13,16]$.

Recall that if $C$ is a smooth curve of the degree $d$ in the projective plane $\mathbb{P}^{2}$, then its genus $g(C)$ is equal to

$$
g(C)=\frac{1}{2}(d-1)(d-2) .
$$

We also have

$$
g(C)=\#\left(\operatorname{int}(\operatorname{Newt}(C)) \cap \mathbb{Z}^{3}\right) .
$$

The set of all curves of the degree $d$ forms projective space of the dimension

$$
\left(\begin{array}{c}
d+2 \\
2
\end{array}\right)-1=\frac{1}{2}(d-1)(d-2)+3 d-1 .
$$


We let $C_{\text {sing }}$ denote a singular curves having $\nu$ knots. Then for its genus we have $g\left(C_{\text {sing }}\right)=\frac{1}{2}(d-1)(d-2)-\nu$.

Now, the question arising is what is the number $N_{g, d}$ of irreducible curves of genus $g$ and degree $d$, which are incident with $g+3 d-1$ general points in a complex projective plane $\mathbb{P}^{2}$. It is remarkable fact that the number $N_{g, d}$ can be found and it can be done by methods of tropical geometry. In particular we have the following theorem, known as the Mikhailkin theorem of correspondence.

Theorem 6 The number of irreducible tropical curves of the degree $d$ and genus $g$ which are incident with $g+3 d-1$ general points in $\mathbb{R}^{2}$, where each curve is counted with multiplicity, is equal to the Gromov-Witten number $N_{g, d}$ of the complex projective plane $\mathbb{P}^{2}$.

In order to illustrate this statement let us list several values of the Gromov-Witten invariants. The simplest Gromov-Witten invariants are $N_{0,1}=1$ and $N_{0,2}=1$. It state that a unique line passes through two points, and that a unique quadric passes through five points. The first non-trivial number is $N_{0,3}=12$. We will explain it briefly. Let curves be defined as cubic polynomials

$$
f=c_{0} x^{3}+c_{1} x^{2} y+c_{2} x^{2} z+c_{3} x y^{2}+c_{4} x y z+c_{5} x z^{2}+c_{6} y^{3}+c_{7} y^{2} z+c_{8} y z^{2}+c_{9} z^{3} .
$$

For general coefficients $c_{0}, c_{1}, \ldots, c_{9}$, the curve $\{f=0\}$ is smooth of genus $g=1$. The curve becomes rational, i.e. the genus drops to $g=0$, precisely when it has a singular point. This is happening if and only if the discriminant of $f$ vanishes. The discriminant $\Delta(f)$ is a homogeneous polynomial of degree 12 in the 10 coefficients $c_{0}, c_{1}, \ldots c_{9}$ which are unknown. It is a sum of 2040 monomials:

$$
\Delta(f)=19683 c_{0}^{4} c_{6}^{4} c_{9}^{4}-26244 c_{0}^{4} c_{6}^{3} c_{7} c_{8} c_{9}^{3}+\cdots-c_{2}^{2} c_{3} c_{4}^{4} c_{5}^{3} c_{6}^{2} .
$$

Discriminants (7) is calculated using [11]. Further, suppose the cubic $\{f=0\}$ is required to pass through eight given points in $\mathbb{P}^{2}$. This translates into eight linear equations in $c_{0}, c_{1}, \ldots c_{9}$. Combining the equation $\Delta(f)=0$ with the eight linear equations, we obtain a system of equations that has 12 solutions in $\mathbb{P}^{9}$. These solutions are the coefficient of the $N_{0,3}=12$ rational cubics we seek.

The other non-trivial numbers are $N_{3,4}=1, N_{2,4}=27, N_{1,4}=255, \quad N_{0,4}=620$. When $g=0$ these values satisfy a nice recurrence relation

$$
N_{0, d}=\sum_{\substack{d_{1}+d_{2}=d \\
d_{1}, d_{2}>0}}\left(d_{1}^{2} d_{2}^{2}\left(\begin{array}{c}
3 d-4 \\
3 d_{1}-2
\end{array}\right)-d_{1}^{3} d_{2}\left(\begin{array}{c}
3 d-4 \\
3 d_{1}-1
\end{array}\right)\right) N_{0, d_{1}} N_{0, d_{2}} .
$$




\subsection{Concluding remarks}

Tropical geometry is a rather new field at the interface between algebraic geometry and combinatorics, and with connections to many other fields. Respectively it is a linear version of algebraic geometry in which polynomials are replaced with piecewise-linear functions, and their zero sets into polyhedral complexes. As such, it is suitable to study using the aid of combinatorics. It has shown that many algebraically difficult operations become easier in the tropical setting, as the structure of the objects seems to be simpler. Because of that tropical geometry has found significant applications, in particular in dynamic programming. In enumerative geometry many open problems are solved using ideas of tropical geometry. It also takes a role in physics, since it appears in the study of the string theory.

\section{Acknowledgement}

I am grateful to the Professors of Dragutin Svrtan and Ivica Martinjak on a topic proposal and great help.

\section{References}

[1] L. Allermann, J. Rau. First Steps in Tropical Intersection Theory, arXiv:0709.3705v3, 2009.

[2] E. Brugallé, I. Itenberg, G. Mikhalkin, K. Shaw, Brief introduction to tropical geometry, arXiv:1502.05950.

[3] D. Cartwright, Tropical complexes, arXiv:1308.3813v2, 2015.

[4] G. Cohen, S. Gaubert, J. P. Quadrat, Duality and separation theorems in idempotent semimodules, Linear Algebra Appl. 379 (2004) 395-422.

[5] J. Depersin, S. Gaubert, M. Joswig, A tropical isoperimetric inequality, Proceedings of the $29^{\text {th }}$ Conference on Formal Power Series and Algebraic Combinatorics (London), Séminaire Lotharingien de Combinatoire 78B (2017) 12 pp.

[6] E. Katz, What is Tropical Geometry, Notices of the AMS, 64 (2017) 380-382.

[7] F. Kirwan, Complex Algebraic Curve, LMS Student Texts 23, Cambridge 1992. 
[8] M. Kontsevich, Y. Manin, Gromov-Witten classes, quantum cohomology, and enumerative geometry, Comm. Math. Phys., 164(3):525-562, 1994.

[9] A. G. Kušnirenko, Newton polyhedra and Bezouts theorem, Funkcional. Anal. i Priložen. 10 (1976) 82-83.

[10] A. Gathmann, Tropical algebraic geometry, Jahresber. Deutsch. Math.-Verein. 108 (2006) 3-32.

[11] Israel M. Gel'fand, M. M. Kapranov, A. V. Zelevinsky, Discriminants, resultants and multidimensional determinants, Birkhäuser, Boston, 2008.

[12] M. Gross, B. Siebert, Logarithmic Gromov-Witten Invariants, J. Amer. Math. Soc. 26 (2013) 451-510.

[13] I. Itenberg, V. Kharlamov, E. Shustin. Welschinger invariant and enumeration of real rational curves, Int. Math. Research Notices, 49 (2003) 2639-2653.

[14] I. Itenberg, G. Mikhalkin, Geometry in the tropical limit, Math. Semesterber., 59 (2012) 57-73.

[15] I. Itenberg, G Mikhalkin, E. Shustin, Tropical Algebraic Geometry, volume 35 of Oberwolfach Seminars Series, Birkhäuser, 2007.

[16] D. Maclagan, B. Sturmfels, Introduction to Tropical Geometry, American Mathematical Society, 2015.

[17] H. Markwig, The enumeration of plane tropical curves, Preprint available at the homepage http://www.mathematik.uni-kl.de/gathmann/pub/hannah.pdf

[18] H. Markwig, Three tropical enumerative problems, Mathematisches Institut, GeorgAugust-Universitt Göttingen: Seminars Winter Term 2007/2008.

[19] G. Mikhalkin, Enumerative Tropical Algebraic Geometry in $\mathbb{R}^{2}$, J. Amer. Math. Soc. 18 (2005) 313-377.

[20] G. Mikhalkin, Tropical geometry and its applications, In International Congress of Mathematicians. Vol. II, pages 827-852. Eur. Math. Soc., Zürich, 2006.

[21] G. Mikhalkin, I. Zharkov, Tropical curves, their Jacobians and Theta functions. Curves and Abelian Varietis, arXiv: 0612.267v2, 2007. 
[22] J. Rabinoff, Tropical analytic geometry, Newton polygons, and tropical intersections, Adv. Math. 229 (2012) 3192-3255.

[23] J. Richter-Gebert, B. Sturmfels, T. Theobald, First Steps in Tropical Geometry, arXiv: 0306.366v2, 2003.

[24] I. Simon, Recognizable sets with multiplicities in the tropical semiring, J. Math. Found. Comput. Sci. (1988) 107-120.

[25] D. Speyer. Tropical Geometry PhD thesis, University of California, Berkeley, 2005.

[26] O. Ya. Viro, From the sixteenth Hilbert problem to tropical geometry, Japan. J. Math. 3 (2008)

[27] O. Ya. Viro, On basic concepts of tropical geometry, Tr. Mat. Inst. Steklova 273 (2011) 271-303. 